EPJ Web of Conferences 49, 10004 (2013)

DOI: $10.1051 /$ epjconf/20134910004

C) Owned by the authors, published by EDP Sciences, 2013

\title{
SUSY vs LHC
}

\author{
Ryuichiro Kitano ${ }^{1, a}$
}

${ }^{1}$ Department of Physics, Tohoku University, Sendai 980-8578, Japan

\begin{abstract}
In light of the discovery of the new particle at $125 \mathrm{GeV}$ and the strong lower limits on the masses of superparticles from LHC, we discuss a possible picture of weak scale supersymmetry.
\end{abstract}

\section{Introduction}

The searches for superparticles at the LHC have been putting severe constraints on weak scale supersymmetry. The lower bound on the gluino mass from searches for jets with missing energy is roughly $[1,2]$,

$$
m_{\tilde{g}} \gtrsim 1-1.5 \mathrm{TeV}
$$

A similar constraint applies for squarks in the first generation. The constraints on the masses of the scalar top and bottom quarks (stop and sbottom) are slightly weaker such as

$$
m_{\tilde{t}, \tilde{b}} \gtrsim 500-600 \mathrm{GeV},
$$

for a non-degenerate neutralino [3, 4].

The new particle at $125 \mathrm{GeV}[5,6]$ is also giving an important constraint on SUSY models. If the particle is interpreted as the lightest Higgs boson in the minimal supersymmetric standard model (MSSM), the stop mass is required to be

$$
m_{\tilde{t}} \gtrsim(\mathrm{a} \mathrm{few}) \times \mathrm{TeV} .
$$

In light of these constraints, we discuss what kind of theoretical framework is implied by the LHC data if SUSY is the solution to the naturalness problem.

\section{The log problem}

In softly broken SUSY models, the quadratic divergence is canceled, and that is supposed to be the solution to the hierarchy problem. As one can see below, that is not quite enough any more. We are facing the situation where one also needs to eliminate the log divergence.

There is a logarithmic divergence in the quantum corrections to mass parameters in supersymmetric standard model. Once we put a large gluino mass, $m_{\tilde{g}}$, at a high scale $M$, the stop mass squared and the quadratic term in the Higgs potential (which is the Higgs mass squared times $(-1 / 2)$ ), receives quantum corrections proportional

\footnotetext{
ae-mail: kitano@tuhep.phys.tohoku.ac.jp
}

to $m_{\tilde{g}}^{2} \log \left(M / m_{\tilde{g}}\right)$. If the log factor is large and if there is no significant cancellation among parameters, we naively expect

$$
m_{h}^{2} \sim m_{\tilde{t}}^{2} \sim m_{\tilde{g}}^{2}
$$

This is clearly inconsistent with $m_{h} \sim 125 \mathrm{GeV}$ and $m_{\tilde{g}} \gtrsim 1 \mathrm{TeV}$. This is the SUSY fine-tuning problem. Especially, in models which are friendly with the grand unification, such as gravity mediation, the log factor tends to be large. In the MSSM where the Higgs mass is obtained by the gauge coupling times the VEV of the Higgs field at tree level, the above relation either predicts an unacceptably large Higgs VEV or light gluino/stop that is no longer allowed by the LHC data.

In order to correct the wrong prediction in Eq. (4), the logarithmic quantum correction should be cut-off at a scale close to the TeV scale. In that case, the size of the quantum corrections are estimated to be

$$
\begin{aligned}
& \delta m_{h}^{2} \sim \frac{y_{t}^{2} N_{c}}{8 \pi^{2}} m_{\tilde{t}}^{2} \sim(0.2)^{2} m_{\tilde{t}}^{2} \\
& \delta m_{\tilde{t}}^{2} \sim \frac{g_{3}^{2}}{4 \pi^{2}} \frac{N_{c}^{2}-1}{N_{c}} m_{\tilde{g}}^{2} \sim(0.3)^{2} m_{\tilde{g}}^{2} .
\end{aligned}
$$

Therefore, a mild hierarchy such as

$$
m_{h} \sim 0.2 m_{\tilde{t}}, \quad m_{\tilde{t}} \sim 0.3 m_{\tilde{g}},
$$

is possible without fine-tuning. For example,

$$
m_{\tilde{g}} \sim 2 \mathrm{TeV}, \quad m_{\tilde{t}} \sim 600 \mathrm{GeV}, \quad m_{h} \sim 120 \mathrm{GeV},
$$

is realized naturally. The spectrum is consistent with the Higgs mass and barely satisfies the LHC searches for superparticles. At this stage, one needs to give up the MSSM since the spectrum is inconsistent with Eq. (3).

Therefore, in light of various news from LHC, a theoretical framework to realize natural SUSY requires:

- the cut-off of the quantum correction at TeV, and

- a new contribution to the Higgs boson mass beyond the stop loop.

Both of the requirements actually say that we should have a new physics beyond the MSSM at the TeV energy scale. 


\section{Naive Dimensional Analysis and Partially Composite Higgs}

One of the most drastic ideas for the $\mathrm{TeV}$ new physics beyond the MSSM is the scenario where the Higgs boson is (partially) a composite particle. A TeV scale strong dynamics is assumed to provide an MSSM like theory as the low energy effective theory. Because of the drastic change of the description at the $\mathrm{TeV}$ scale, one may expect a cutoff of the log divergence and a new contribution to the Higgs mass, simultaneously.

In fact, modern efforts of SUSY model building have started with this type of models [7-9]. SUSY is introduced to protect the quadratic divergence of the Higgs potential whereas the electroweak scale is generated by some dynamics at $\mathrm{TeV}$ as in the technicolor model. Such models are now well motivated to be considered again given that there is a light Higgs boson which is not quite as light as the MSSM predictions.

Once we assume that there is some dynamics at a scale $\Lambda$ responsible for the electroweak symmetry breaking, the naive dimensional analysis [10,11] says

$$
\Lambda \sim 4 \pi v \sim 3 \mathrm{TeV}, \quad m_{h} \sim \Lambda .
$$

where $v=246 \mathrm{GeV}$. This is the typical prediction of the technicolor models. Now, for having a light Higgs boson, one can assume that the Higgs fields are slightly weakly coupled to the dynamics at the scale $\Lambda$. By introducing a dimensionless parameter $\epsilon$ which measures the weakness of the coupling compared to the naive estimates, we have

$$
\Lambda \sim 4 \pi \epsilon v, \quad m_{h} \sim \epsilon \Lambda \sim m_{H},
$$

where $m_{H}$ is the size of the soft SUSY breaking contribution to the Higgs potential. In the MSSM language, $\epsilon \Lambda$ is the $\mu$-term and $m_{H}$ is the soft SUSY breaking mass. From Eq. (10), we have

$$
\frac{m_{h}}{v} \sim 4 \pi \epsilon^{2}
$$

By putting $m_{h}=125 \mathrm{GeV}$, we obtain $\epsilon \sim 0.2$ and $\Lambda \sim$ $600 \mathrm{GeV}$. If the top quark is also involved in the dynamics, the estimate of the dynamically generated top quark mass is

$$
m_{t} \sim \epsilon_{t}^{2} \Lambda
$$

where $\epsilon_{t}$ again measures the weakness of the coupling to the strong sector. For $m_{t} \sim 170 \mathrm{GeV}$, we have

$$
\epsilon_{t} \sim 0.5
$$

From these estimates, we have a rough picture:

- there is some dynamics at $\Lambda \sim 600 \mathrm{GeV}$,

- the Higgs fields (and possibly the top quark) are weakly coupled to the dynamics with a suppression factor $\epsilon \sim$ 0.2 (and $\epsilon_{t} \sim 0.5$ ), and

- above the scale $\Lambda$, the picture drastically changes so that the log divergences of the soft SUSY breaking parameters are cut-off.
The Higgs quartic coupling and the Higgsino mass are both dominated by the dynamically generated potential. This is very different from the MSSM case where the Higgs potential is mainly from the $S U(2)_{L} \times U(1)_{Y}$ gauge interactions ( $D$-term) and the $\mu$-term is added by hand.

The parameters $\epsilon$ and $\epsilon_{t}$ can be thought of as the degree of compositeness. The relations among $v, m_{h}$, and $m_{t}$ suggest that the Higgs/top sector is not fully composite, but maybe partially composite [12]. The partially composite scenario is realized only when there is some reason for the absence of the tree-level Higgs potential of $O(\Lambda)$. SUSY is giving a good reason for it since one can naturally have an approximately flat potential even at the quantum level. The (approximately) massless composite particle is ubiquitous in SUSY gauge theories.

\section{A model for dynamical electroweak symmetry breaking}

A pretty simple model of partially composite Higgs can be constructed $[13,14]$. The dynamical sector is an $\mathcal{N}=1$ SUSY $U(2)$ gauge theory with five flavors of chiral superfields. The assignment of the quantum numbers of the standard model gauge group, $(S U(3), S U(2))_{Y}$, for the matter fields is

$$
\begin{aligned}
& f_{A}:(1,2)_{0}, \quad \bar{f}_{A}:(1,1)_{ \pm 1 / 2}, \quad(A=1,2), \\
& f_{\alpha}^{\prime}:(3,1)_{1 / 6}, \quad \bar{f}_{\alpha}^{\prime}:(\overline{3}, 1)_{-1 / 6}, \quad(\alpha=3,4,5) .
\end{aligned}
$$

This theory is in the conformal window [15]. The Higgs field, $H=\left(H_{u}, H_{d}\right)$, and the top quark, $q, t^{c}$, can couple to this CFT through the superpotential terms:

$$
W=\lambda_{H} \bar{f} H f+\lambda_{q} \bar{f}^{\prime} q f+\lambda_{t} \bar{f} t^{c} f^{\prime} .
$$

The gauge coupling $g$ and the coupling constants $\lambda_{H}, \lambda_{q}$, and $\lambda_{t}$ flow to IR fixed points whose values are estimated by the $a$-maximization [16] such as:

$$
\begin{aligned}
& \frac{g}{4 \pi} \sim 0.4, \quad \frac{\lambda_{H_{d}}}{4 \pi} \sim 0.1, \quad \frac{\lambda_{H_{u}}}{4 \pi} \sim 0.3, \\
& \frac{\lambda_{q}}{4 \pi} \sim 0.3, \quad \frac{\lambda_{t}}{4 \pi} \sim 0.3 .
\end{aligned}
$$

By adding a mass to $f^{\prime}$ and $\bar{f}^{\prime}$ by superpotential:

$$
W=\Lambda \bar{f}^{\prime} f^{\prime}
$$

the $S U(2)$ gauge interaction becomes strong and confines at the scale $\Lambda$. The coupling constants $\lambda$ 's receive multiplicative renormalization, $\lambda \rightarrow \lambda k$ with $k>1$. By turning on SUSY breaking terms by

$$
\Lambda \rightarrow \Lambda\left(1+m_{\mathrm{SUSY}}^{2} \theta^{2}\right)
$$

the Higgs potential are generated. For $\Lambda \sim m_{\mathrm{SUSY}}$, the lightest Higgs field (which is mainly $H_{d}$ ) obtain a mass:

$$
\left.m_{h}^{2} \sim\left(\frac{\lambda_{H_{d}}}{4 \pi}\right)^{2} \Lambda^{2} \sim m_{h}^{2}\right|_{\mathrm{MSSM}}+\frac{\lambda_{H_{d}}^{4} v^{2}}{(4 \pi)^{2}},
$$


and the Higgsino mass is also generated as $m_{\tilde{H}} \sim$ $\left(\lambda_{H_{u}} \lambda_{H_{d}} /(4 \pi)^{2}\right) \Lambda$. The dynamically generated top quark mass is

$$
m_{t} \sim \frac{\lambda_{q} \lambda_{t} \lambda_{H_{d}}}{(4 \pi)^{2}} v
$$

The correspondence to discussion in the previous section is

$$
\epsilon \sim \frac{\lambda_{H_{d}}}{4 \pi}, \quad \epsilon_{t}^{2} \sim \frac{\lambda_{q} \lambda_{t}}{(4 \pi)^{2}} .
$$

We see that the required value $\epsilon \sim 0.2$ and $\epsilon_{t}^{2} \sim(0.5)^{2}$ is roughly consistent with the fixed point values with the $k$ factor $\sim 2$.

This model should be thought of as the effective theory around the scale $\Lambda$. In order to cut-off the log divergence proportional to the gluino mass squared, the semi-weakly coupled description above should be replaced by a more strongly coupled one. For example, one can assume that the gauge symmetry is extended to $S U(3)$ above the scale $\Lambda$, which makes the theory a strongly coupled CFT. In this case, the stop mass parameter is also strongly renormalized such as

$$
\frac{d}{d \log \mu} m_{\tilde{t}}^{2}=c m_{\tilde{t}}^{2}-\frac{g_{3}^{2}}{(4 \pi)^{2}} \frac{N_{c}^{2}-1}{N_{c}} m_{\tilde{g}}^{2}+\cdots,
$$

where $c$ is of $O(1)$. The quasi IR fixed point of the stop mass is then roughly

$$
m_{\tilde{t}}^{2} \sim \frac{g_{3}^{2}}{(4 \pi)^{2} c} \frac{N_{c}^{2}-1}{N_{c}} m_{\tilde{g}}^{2},
$$

which is equivalent to estimate without the log divergence. The same is true for the effect of the stop mass to the Higgs mass parameters. One can consider a more drastic change of the theory such as the appearance of an extra dimension at a scale $\Lambda$. See Ref. [14] for a proposal to obtain composite Higgs fields couple to the $U(2)$ model above from a higher dimensional QCD.

\section{Why SUSY?}

We see that there is a simple solution to the SUSY finetuning problem once we cut-off the theory at the TeV scale. But once we give up the SUSY desert, why do we need SUSY?

Aside from the theoretical beauty or calculability, we should remember that the presence of the light Higgs boson already provides a good motivation. The naive dimensional analysis says that relation between the decay constant $v$ and the mass of the $0^{+}$resonance, $m_{h}$, is estimated to be

$$
m_{h} \sim 4 \pi v \sim 3 \mathrm{TeV},
$$

which is quite different from the situation in the electroweak sector. On the other hand, the MSSM provides a relation

$$
m_{h} \sim m_{Z} \ll 4 \pi v .
$$

Although this turns out to be too small, SUSY provides an excellent starting point to explain $125 \mathrm{GeV} ; m_{Z} \sim 91 \mathrm{GeV}$ rather than $3 \mathrm{TeV}$.

\section{Lessons from QCD}

The QCD is a natural place to look for a hint for the theory of electroweak symmetry breaking. Other than the electroweak, that is the only known example of the spontaneous symmetry breaking, actually happening in the vacuum. Indeed, the patterns of the symmetry breaking in the chiral symmetry breaking and the electroweak symmetry breaking are identical; $S U(2)_{L} \times U(1)_{Y} \rightarrow U(1)_{\mathrm{EM}}$. The only big difference is the mass of $0^{+}$resonances, i.e., the Higgs boson mass relative to the order parameter $v$ or $f_{\pi}$.

When we look up the table in the PDG [17], we actually find relatively light scalar mesons:

$$
f_{0}(600), \quad f_{0}(980)
$$

in which $f_{0}(600)$ is a pretty broad resonance. These are the candidates of the Higgs boson counterparts in QCD. There are also light and narrow vector resonances

$$
\rho(770), \quad \omega(782) \text {. }
$$

Of course, there are pions, $\pi^{0}$ and $\pi^{ \pm}$associated with the chiral symmetry breaking, corresponding to the longitudinal modes of $Z^{0}$ and $W^{ \pm}$in the electroweak symmetry breaking.

One can construct an effective theory to describe the interactions among these light hadrons as the Higgs model [18, 19], which is the (linearly realized) Hidden Local Symmetry [20]. The model is based on a $U(2)$ gauge theory where we introduce Higgs fields:

$$
H_{L}^{i A}, \quad H_{R}^{i A}, \quad(i, A=1,2) .
$$

Both are $2 \times 2$ matrices. One of the indices is for $U(2)$ gauge group and the other is for flavor symmetry, $S U(2)_{L} \times$ $S U(2)_{R}$. The $H_{L}$ and $H_{R}$ fields transform under $S U(2)_{L}$ and $S U(2)_{R}$, respectively. By requiring the flavor symmetry in the Lagrangian and setting up the potential so that the minimum is at

$$
H_{L}=H_{R} \propto \mathbf{1},
$$

the chiral symmetry is spontaneously broken down to a diagonal group, and provides massless pions. The radial direction corresponds to the Higgs boson, $f_{0}(980)$ (or $\left.f_{0}(600)\right)$, and the $U(2)$ gauge fields obtain masses which correspond to $\rho(770)$ and $\omega(782)$.

This simple linear sigma model contains a pretty interesting object, a string associated with the gauge symmetry breaking. By setting the Lagrangian parameters so that the masses and the couplings of hadrons are reproduced, one can estimate the tension of the string as [19]:

$$
\sigma \sim(400 \mathrm{MeV})^{2} .
$$

In QCD, there is a natural object to be identified with this string. It is the QCD string which stretches between color sources to cause confinement. The tension of the QCD string is estimated by the lattice simulations and also from the quarkonium spectrum, and both give approximately $\sigma \sim(430 \mathrm{MeV})^{2}$, which is very consistent with Eq. (32). 
If the string in the linear sigma model corresponds to the QCD string, there appears an interesting interpretation of the Hidden Local Symmetry. The QCD string is supposed to carry the color flux whereas the string in the sigma model is carrying the magnetic flux of the $\rho$ and $\omega$ mesons. The identification then says that the $\rho$ and $\omega$ mesons are the magnetic gauge bosons in QCD. Since the Higgs fields $H_{L}$ and $H_{R}$ are charged under $U(2)$, they are (non-abelian) magnetic monopoles. The Higgs phenomena of the magnetic gauge theory, that is also describing the chiral symmetry breaking, is now identified as the color confinement in QCD.

There is a theoretical support for this interpretation. It has been demonstrated in Ref. [18], that by making a deformation of a SUSY QCD model, one can see that the Hidden Local Symmetry appears as the magnetic theory via the Seiberg duality. See also earlier discussion [2123 ] on the interpretation of the $\rho$ meson as the magnetic gauge boson.

The Seiberg dual picture of QCD is a $U(2)$ gauge theory with five flavors, that is in fact identical to the model discussed in Section 4 for the model of dynamical electroweak symmetry breaking. The Higgs fields introduced above, $H_{L}$ and $H_{R}$ correspond to $f$ and $\bar{f}$, respectively, in the model of Section 4 which arise as dual quarks transforming under both the magnetic gauge group and the flavor group. By adding soft SUSY breaking terms, the dual quarks obtain VEVs and the magnetic gauge group is Higgsed while the chiral symmetry breaking happens. This Higgs mechanism in the magnetic picture corresponds to the confinement.

Interestingly, a model of the low energy QCD can be regarded as the magnetic picture, and the magnetic picture has the same structure as the model of partially composite Higgs for the electroweak symmetry breaking. From this view, physics behind the electroweak symmetry breaking may actually be very similar to (or the same as) QCD, and the quantitative difference, the mass of the Higgs boson, may originate simply from whether SUSY is a good (approximate) symmetry at the dynamical scale.

\section{Predictions}

There are two kinds of predictions in the framework we discussed. One is the SUSY spectrum in Eq. (8). Since these are the estimates from (probably unavoidable) finite quantum corrections, the naturalness suggests that the estimates roughly give the upper bounds. The light stop should be observed at the LHC soon.

Another interesting prediction is the presence of the relatively light resonances from the strong sector. As we have discussed, the light Higgs fields actually suggest a low dynamical scale such as $\Lambda \sim 600 \mathrm{GeV}$, from the naive dimensional analysis. Since the dynamics should have $S U(2)_{L} \times U(1)_{Y}$ as a part of the global symmetry, one expects vector resonances, $W^{\prime}$ and $Z^{\prime}$, associated with the current operators of the global symmetry. These are the $\rho$ meson counterparts of QCD. In the explicit model of Section 4 , they corresponds to $U(2)$ gauge bosons. Finding such resonances will be very important for the understanding of the physics of electroweak symmetry breaking.

\section{Summary}

The limits on SUSY particles from LHC and the $125 \mathrm{GeV}$ new particle may be suggesting a very interesting possibility, a dynamical electroweak symmetry breaking much below $4 \pi v \sim 3 \mathrm{TeV}$. In such a case, we will see many resonances and also superparticles at the LHC, opening up a new era of particle physics to look for the theory behind the electroweak symmetry breaking, just as it has been done to look for QCD since the days of the discovery of the pions.

\section{Acknowledgments}

I would like to thank organizers of HCP2012. This work is supported in part by the Grant-in-Aid for Scientific Research 23740165 of JSPS.

\section{References}

[1] The ATLAS Collaboration, ATLAS-CONF-2012109.

[2] S. Chatrchyan et al. [CMS Collaboration], Phys. Rev. Lett. 109, 171803 (2012) [arXiv:1207.1898 [hep-ex]].

[3] The ATLAS Collaboration, ATLAS-CONF-2013001, ATLAS-CONF-2012-165.

[4] The CMS Collaboration, CMS PAS SUS-12-023, CMS PAS SUS-12-028.

[5] G. Aad et al. [ATLAS Collaboration], Phys. Lett. B 716, 1 (2012) [arXiv:1207.7214 [hep-ex]].

[6] S. Chatrchyan et al. [CMS Collaboration], Phys. Lett. B 716, 30 (2012) [arXiv:1207.7235 [hep-ex]].

[7] E. Witten, Nucl. Phys. B 188, 513 (1981).

[8] M. Dine, W. Fischler and M. Srednicki, Nucl. Phys. B 189, 575 (1981).

[9] S. Dimopoulos and S. Raby, Nucl. Phys. B 192, 353 (1981).

[10] A. Manohar and H. Georgi, Nucl. Phys. B 234, 189 (1984).

[11] M. A. Luty, Phys. Rev. D 57, 1531 (1998) [hep$\mathrm{ph} / 9706235]$.

[12] R. Kitano, M. A. Luty and Y. Nakai, JHEP 1208, 11 (2012) [arXiv:1206.4053 [hep-ph]].

[13] H. Fukushima, R. Kitano and M. Yamaguchi, JHEP 1101, 111 (2011) [arXiv:1012.5394 [hep-ph]].

[14] R. Kitano and Y. Nakai, arXiv:1212.2726 [hep-ph].

[15] N. Seiberg, Nucl. Phys. B 435, 129 (1995) [hepth/9411149].

[16] K. A. Intriligator and B. Wecht, Nucl. Phys. B 667, 183 (2003) [hep-th/0304128].

[17] J. Beringer et al. [Particle Data Group Collaboration], Phys. Rev. D 86, 010001 (2012).

[18] R. Kitano, JHEP 1111, 124 (2011) [arXiv:1109.6158 [hep-th]]. 
[19] R. Kitano, M. Nakamura and N. Yokoi, Phys. Rev. D 86, 014510 (2012) [arXiv:1202.3260 [hep-ph]].

[20] M. Bando, T. Kugo, S. Uehara, K. Yamawaki and T. Yanagida, Phys. Rev. Lett. 54, 1215 (1985).

[21] N. Seiberg, Int. J. Mod. Phys. A 16, 4365 (2001) [hep-th/9506077].
[22] M. Harada and K. Yamawaki, Phys. Rev. Lett. 83, 3374 (1999) [hep-ph/9906445].

[23] Z. Komargodski, JHEP 1102, 019 (2011) [arXiv:1010.4105 [hep-th]]. 